\title{
Pyriform Fossa Carcinoma
}

National Cancer Institute

\section{Source}

National Cancer Institute. Pyriform Fossa Carcinoma. NCI Thesaurus. Code C6700.

A carcinoma that arises from the pyriform sinus. Patients usually present with advanced stage disease and the prognosis is poor. 